\title{
DECIMETER-WAVELENGTH STUDIES OF RECOMBINATION LINES
}

\author{
YERVANT TERZIAN and VERNON PANKONIN \\ National Astronomy and Ionosphere Center, Cornell University, Ithaca, N.Y., U.S.A.
}

\begin{abstract}
The results of new studies of decimeter wavelength $\mathrm{Hn} \alpha$ and $\mathrm{Cn} \alpha$ lines toward galactic nebulae are reported. The observations were made with the 305-m radio telescope of the Arecibo Observatory at $430 \mathrm{MHz}(247 \alpha$ and $248 \alpha)$ and $606 \mathrm{MHz}(221 \alpha)$. Selected positions in W51, W49, and the Rosette nebula were observed. The observed lines exhibit no pressure broadening; however, this is consistent with the interpretation of the data.
\end{abstract}

\section{Introduction}

Observations of radio recombination lines are well established as an important means of studying the properties of $\mathrm{H}$ II regions, especially when a nebula is optically obscured. However, present analyses of hydrogen lines are based almost exclusively on data obtained at frequencies greater than $\sim 1 \mathrm{GHz}$. Low frequency observations are necessary in order to determine whether the source parameters derived can account for hydrogen line emission characteristics over an extended frequency range as well.

Current theories of pressure broadening in recombination lines predict a very strong dependence on the principal quantum number of the transition. Therefore, low frequency (high quantum number $n$ ) observations seem to be ideal tests for the significance of these effects.

Recombination line observations also promise to provide information which is vital to an understanding of the physical processes in predominantly neutral hydrogen regions. In this case the factors which influence the recombination line emission are not well known, and observational data are relied upon to indicate the types of source models which must be considered. In particular there are no convincing predictions of the frequency behavior of carbon recombination emission, but one interesting fact was noticed: The carbon lines become stronger relative to the corresponding hydrogen lines as the frequency of the observations decreases. The behavior of carbon line emission at frequencies below $1 \mathrm{GHz}$ must be determined experimentally to help limit the possible source models.

Successful recombination line observations have been performed at frequencies below $\sim 0.7 \mathrm{GHz}$. Penfield et al. (1967) reported the detection of $\mathrm{H} 253 \alpha(\sim 0.4 \mathrm{GHz})$, but only from W80. Pedlar and Davies (1972) have obtained $220 \alpha(\sim 0.61 \mathrm{GHz})$ spectra toward 12 positions in galactic $\mathrm{H}_{\mathrm{II}}$ regions. In the present paper we will outline the major results of observations at the Arecibo observatory which extend the frequency range to $0.43 \mathrm{GHz}(248 \alpha)$ for several selected nebulae (Parrish et al., 1972; Parrish et al., 1974; Pankonin and Parrish, 1974). 


\section{Observations at Arecibo}

To date recombination line observations at Arecibo have only been performed in the lines of sight to known $\mathrm{H}$ II regions. $247 \alpha$ and/or $248 \alpha(\sim 0.43 \mathrm{GHz})$ and $221 \alpha$ $(\sim 0.61 \mathrm{GHz})$ spectra were obtained toward five positions in three well-known galactic nebulae. The relevant instrumental parameters are given in Table I. The positions observed are listed in Table II together with the hydrogen line parameters derived from these observations. (The $247 \alpha$ and $248 \alpha$ spectra were averaged before the line parameters were determined.) The parameters of detected carbon lines are listed in Table III. The 247, 248 $\alpha$ spectra of W51 $A$ and W49 $A$ are shown in Figures 1 and 2.

The intensity of helium recombination emission from $\mathrm{H}$ II regions is expected to be $\sim 10 \%$ that of the corresponding hydrogen line (Palmer et al., 1969; and Churchwell, 1970). There was no indication of helium lines in any of the $221 \alpha$ and $247,248 \alpha$ spectra observed at Arecibo (Pankonin, 1973). However, since the peak intensities of the hydrogen lines in this study were $\lesssim 10$ times the rms noise in the spectra this result is

TABLE I

Instrumental parameters

\begin{tabular}{llllll}
\hline $\begin{array}{l}\text { Frequency } \\
(\mathrm{MHz})\end{array}$ & Transition & $\begin{array}{l}\text { Sensitivity } \\
\left(\mathrm{K} \mathrm{Jy}^{-1}\right)\end{array}$ & $\begin{array}{l}\text { HPBW } \\
(\mathrm{arcmin})\end{array}$ & $\begin{array}{l}\text { System temp. } \\
(\mathrm{K})\end{array}$ & $\begin{array}{l}\text { Max. freq. resol. } \\
\left(\mathrm{km} \mathrm{s}^{-1}\right)\end{array}$ \\
\hline 606 & $221 \alpha$ & 4.5 & 9 & 170,350 & $3.3^{\mathrm{a}}$ \\
430 & $247 \alpha, 248 \alpha$ & 14.7 & 9 & 130 & $4.8^{\mathrm{a}}$ \\
\hline
\end{tabular}

a These correspond to $7 \mathrm{kHz}$.

TABLE II

Hn $\alpha$ Observations

\begin{tabular}{|c|c|c|c|c|c|}
\hline Source & W51 $A$ & W51 $B$ & W51C & $\mathrm{W} 49 A$ & Rosette \\
\hline Gal. Coord. & G49.5-0.4 & $\begin{array}{l}G 49.0-0.3 \\
G 49.1-0.4\end{array}$ & $\mathrm{G} 49.0-0.6$ & $\mathrm{G} 43.2+0.0$ & G206.0-2.1 \\
\hline R.A. (1950) & $19^{\mathrm{h}} 21^{\mathrm{m}} 28^{\mathrm{s}}$ & $19^{\mathrm{h}} 20^{\mathrm{m}} 18^{\mathrm{s}}$ & $19^{\mathrm{h}} 21^{\mathrm{m}} 25^{\mathrm{s}}$ & $19^{\mathrm{h}} 07^{\mathrm{m}} 52^{\mathrm{s}}$ & $06^{\mathrm{h}} 28^{\mathrm{m}} 30^{\mathrm{s}}$ \\
\hline Dec. $(1950)$ & $14^{\circ} 24^{\prime} 28^{\prime \prime}$ & $14^{\circ} 02^{\prime} 05^{\prime \prime}$ & $13^{\circ} 52^{\prime} 00^{\prime \prime}$ & $08^{\circ} 59^{\prime} 08^{\prime \prime}$ & $05^{\circ} 13^{\prime} 20^{\prime \prime}$ \\
\hline \multicolumn{6}{|c|}{$\underline{\mathrm{H} 247,248 \alpha}$} \\
\hline $\begin{array}{l}T_{\mathrm{L}} / T_{\mathrm{c}} \\
\Delta v_{\mathrm{L}}\left(\mathrm{km} \mathrm{s}^{-1}\right) \\
V_{\mathrm{LSR}}\left(\mathrm{km} \mathrm{s}^{-1}\right)\end{array}$ & $\begin{array}{l}(1.8 \pm 0.1) 10^{-3} \\
31.4 \pm 2.3 \\
54.6 \pm 0.9\end{array}$ & $\begin{array}{l}(3.7 \pm 0.4) 10^{-3} \\
30.9 \pm 3.7 \\
64.1 \pm 1.6\end{array}$ & $\begin{array}{l}(3.2 \pm 0.8) 10^{-3} \\
15.3 \pm 4.1 \\
56.3 \pm 2.4\end{array}$ & $\begin{array}{l}(1.8 \pm 0.2) 10^{-3} \\
15.0 \pm 1.7 \\
11.2 \pm 0.8\end{array}$ & $\begin{array}{l}(2.8 \pm 0.3) 10^{-3} \\
22.1 \pm 3.2 \\
10.8 \pm 1.3\end{array}$ \\
\hline \multicolumn{6}{|c|}{$\underline{\mathrm{H} 221 \alpha}$} \\
\hline $\begin{array}{l}T_{\mathrm{L}} / T_{\mathrm{c}} \\
\Delta v_{\mathrm{L}}\left(\mathrm{km} \mathrm{s}^{-1}\right) \\
V_{\mathrm{LSR}}\left(\mathrm{km} \mathrm{s}^{-1}\right)\end{array}$ & $\begin{array}{l}(1.9 \pm 0.2) 10^{-3} \\
31.6 \pm 3.2 \\
58.9 \pm 1.4\end{array}$ & $\begin{array}{l}(3.9 \pm 0.5) 10^{-3} \\
32.8 \pm 4.5 \\
58.4 \pm 1.8\end{array}$ & $\begin{array}{l}<3.0 \times 10^{-3} \\
-\end{array}$ & $\begin{array}{l}(3.4 \pm 0.5) 10^{-3} \\
8.4 \pm 1.4 \\
9.7 \pm 0.7\end{array}$ & $\begin{array}{l}(4.8 \pm 0.6) 10^{-3} \\
17.2 \pm 2.3 \\
15.1 \pm 1.0\end{array}$ \\
\hline
\end{tabular}

Note: Errors quoted here and in Table III represent one standard deviation. 
TABLE III

$\mathrm{Cn} \alpha$ Observations

\begin{tabular}{|c|c|c|c|}
\hline Source & W51 $A$ & $\mathrm{~W} 51 B$ & $\mathrm{~W} 49 A^{\mathrm{a}}$ \\
\hline & & $\mathrm{C} 247,248 \alpha$ & \\
\hline $\begin{array}{l}T_{\mathrm{L}} / T_{\mathrm{c}} \\
\Delta v_{\mathrm{L}}\left(\mathrm{km} \mathrm{s}^{-1}\right) \\
V_{\mathrm{LSR}}\left(\mathrm{km} \mathrm{s}^{-1}\right)\end{array}$ & $\begin{array}{l}(1.4 \pm 0.1) 10^{-3} \\
15.2 \pm 1.8 \\
61.6 \pm 0.8\end{array}$ & $\begin{array}{l}(2.2 \pm 0.7) 10^{-3} \\
8.4 \pm 3.6 \\
62.2 \pm 1.6\end{array}$ & $\begin{array}{l}\leqslant 1.0 \times 10^{-3} \\
- \\
-\end{array}$ \\
\hline$V_{\mathrm{LSR}}\left(\mathrm{km} \mathrm{s}^{-1}\right)$ & & $\mathrm{C} 221 \alpha$ & \\
\hline $\begin{array}{l}T_{\mathrm{L}} / T_{\mathrm{c}} \\
\Delta v_{\mathrm{L}}\left(\mathrm{km} \mathrm{s}^{-1}\right) \\
V_{\mathrm{LSR}}\left(\mathrm{km} \mathrm{s}^{-1}\right)\end{array}$ & $\begin{array}{l}(1.1 \pm 0.2) 10^{-3} \\
16.2 \pm 3.7 \\
62.0 \pm 1.8\end{array}$ & $\begin{array}{l}\leqslant 2.4 \times 10^{-3} \\
-\end{array}$ & $\begin{array}{l}- \\
- \\
-\end{array}$ \\
\hline
\end{tabular}

a A recombination line has been detected in the spectrum of $\mathrm{W} 49 \mathrm{~A}$, which may be due to carbon and is probably not associated with the W $49 A$ source; see text for discussion.

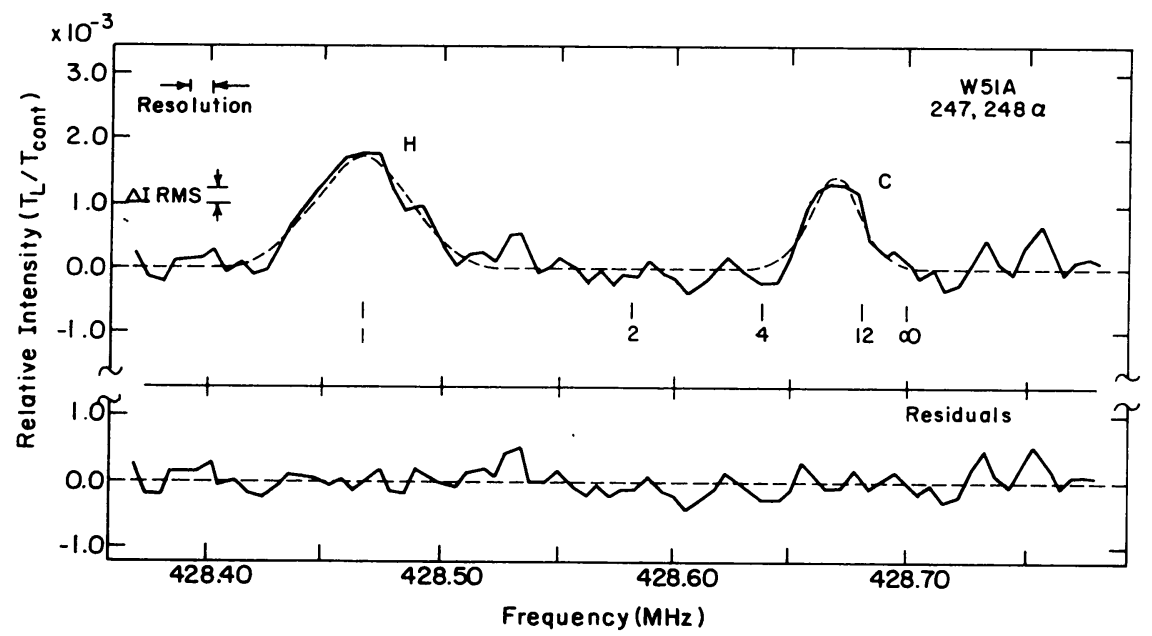

Fig. 1. 247, 248 $\alpha$ spectrum of W51 $A$ and the residuals after subtraction of the fitted function (dashed line in the upper part). The numbers beneath the spectrum indicate the expected position of recombination lines of hydrogenic emitters of that mass if they have the same radial velocity as the $\mathrm{H}$ line.

not surprising. Pedlar and Davies (1972) conclude that in certain $\mathrm{H}_{\text {II }}$ regions there may be a deficiency of ionized helium in the low density parts of the nebulae which are responsible for the major contribution to the low frequency hydrogen lines. This would imply that the Strömgren spheres of hydrogen and singly ionized helium do not coincide. 


\section{The Hn $\alpha$ Lines}

The general problem in interpreting the low frequency hydrogen line observations is to explain the frequency dependence of the emission between $221 \alpha$ and $248 \alpha$. The integrated line intensity at $248 \alpha$ relative to $221 \alpha$ was usually larger than could be explained by assuming uniform (in density and temperature) source models. A density and/or temperature distribution in the source or nonthermal continuum radiation in

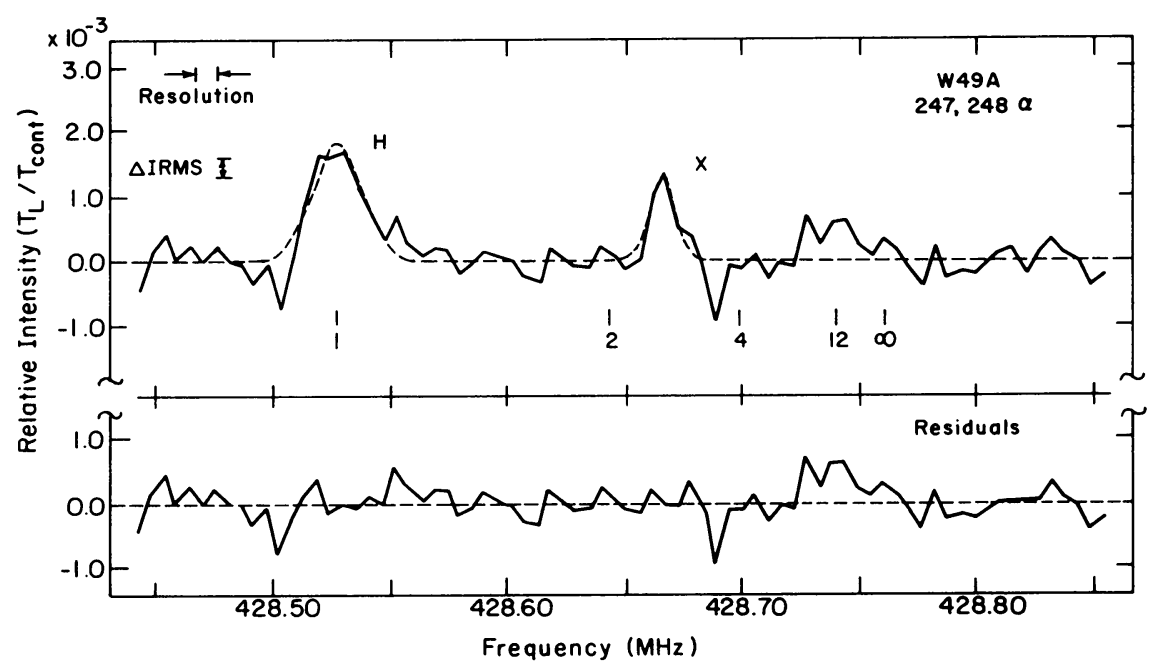

Fig. 2. 247, $248 \alpha$ spectrum of W49A. Figure format is described in the Figure 1 caption.

the beam provides the most straightforward explanation of the observed behavior. (The latter applies to W51C.)

In analyzing the low frequency results it is not possible to assume a priori either an optically thin source or LTE emission. Both optical depth and LTE departure effects must be considered, and both are functions of the frequency and the density and temperature of the source. With the limited amount of data available at low frequencies it is possible to infer approximate source models which will account for the observed emission, and thus to estimate the electron temperature and density of the emitting regions.

The electron temperatures for the $\mathrm{H}$ il regions considered here are fairly well known and fall within the range $5000 \lesssim T_{\mathrm{e}} \lesssim 10000 \mathrm{~K}$. These temperatures are typical of $\mathrm{H}$ II regions in general. Normally the density structure is more pronounced than temperature variations in $\mathrm{HII}$ regions, hence to a first approximation isothermal source models can be assumed. Only in the case of W49 $A$ must there be a temperature distribution as well as a density distribution.

The Doppler temperature, calculated from the measured width of a Doppler broadened line, can be considered an upper limit to the electron temperature in the emitting region. In this way the widths of the $H 247,248 \alpha$ and $H 221 \alpha$ lines from W49 $A$ 
impose an upper limit to the electron temperature of $\sim 2700 \mathrm{~K}$ (see Figure 4 ) in the part of the nebula which emits those low frequency lines.

If current pressure broadening theories are correct, it should be possible to use the measured widths of low frequency recombination lines to estimate the electron density in the emitting region, or at least obtain an upper limit. Griem (1967) indicates pressure broadening effects are very sensitive to the principal quantum level of the transition and should dominate the line width for high level transitions. For the ratio of the pressure broadened to Doppler broadened line width, Griem gives

$$
\frac{\Delta v_{\mathrm{p}}}{\Delta v_{\mathrm{D}}} \propto \frac{N_{\mathrm{e}}}{\sqrt{T_{\mathrm{e}} T_{\mathrm{D}}}} n^{7},
$$

where $T_{\mathrm{e}}$ and $T_{\mathrm{D}}$ are the electron and Doppler temperatures and $N_{\mathrm{e}}$ is the electron density of the emitting region.

There was no conclusive evidence of pressure broadening in any of the lines observed at Arecibo (see also Parrish et al., 1972). Figures 3 and 4 show the measured

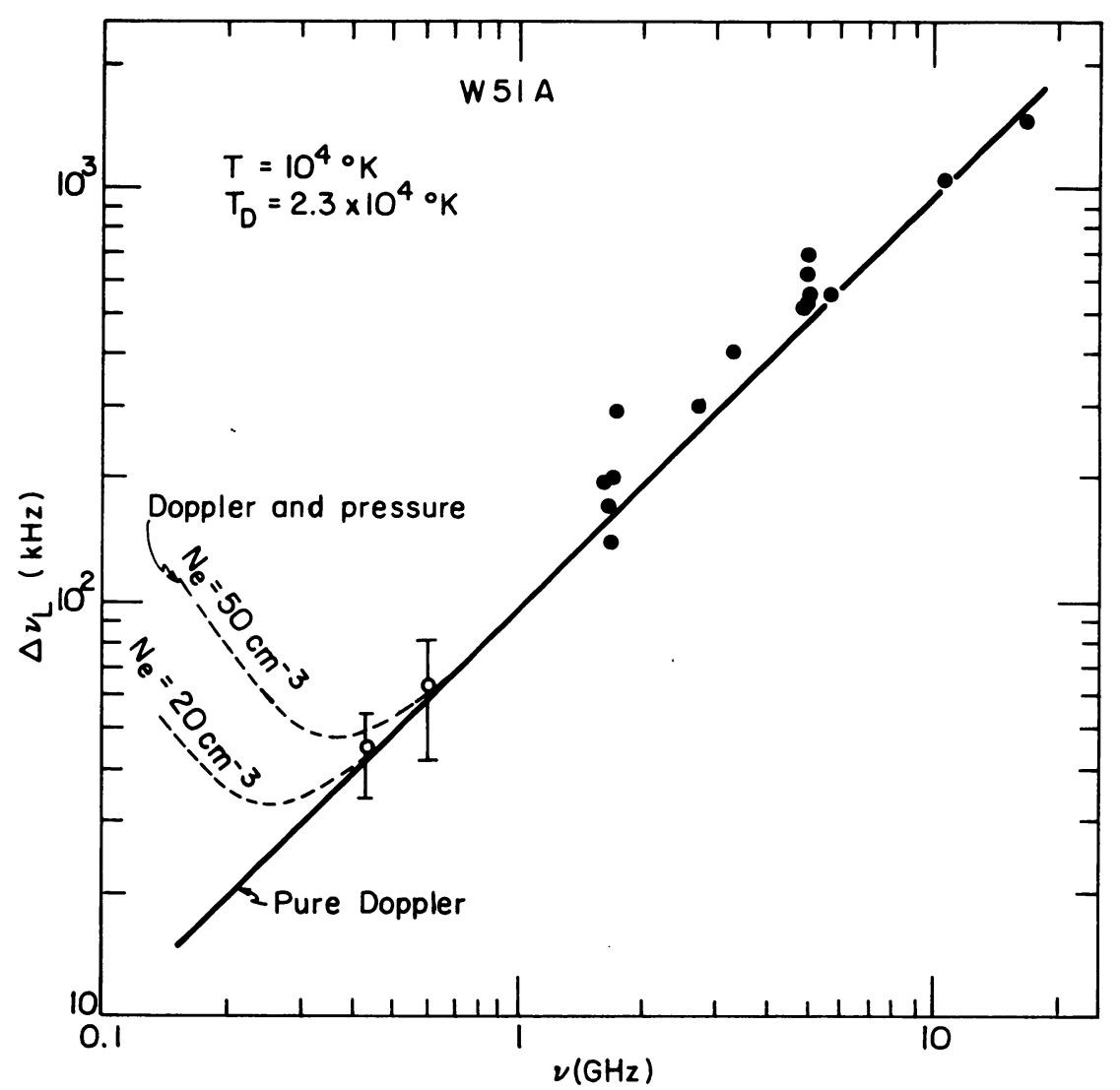

Fig. 3. Widths of $\mathrm{Hn} \alpha$ lines from W51 $A$. Open circles indicate present results. Pressure broadening effects (dashed curves) are compared to expected pure Doppler broadened line widths for the physical conditions indicated. 
widths of $\mathrm{Hn} \alpha$ lines plotted vs. frequency of the observations from W51 $A$ and W49A. In W $49 A$ the low frequency line widths are narrower than expected for Doppler broadening, in part indicating low temperatures as discussed above. It should be pointed out that the attenuation of the line radiation by continuum absorption increases with decreasing frequency, and pressure broadening redistributes the line

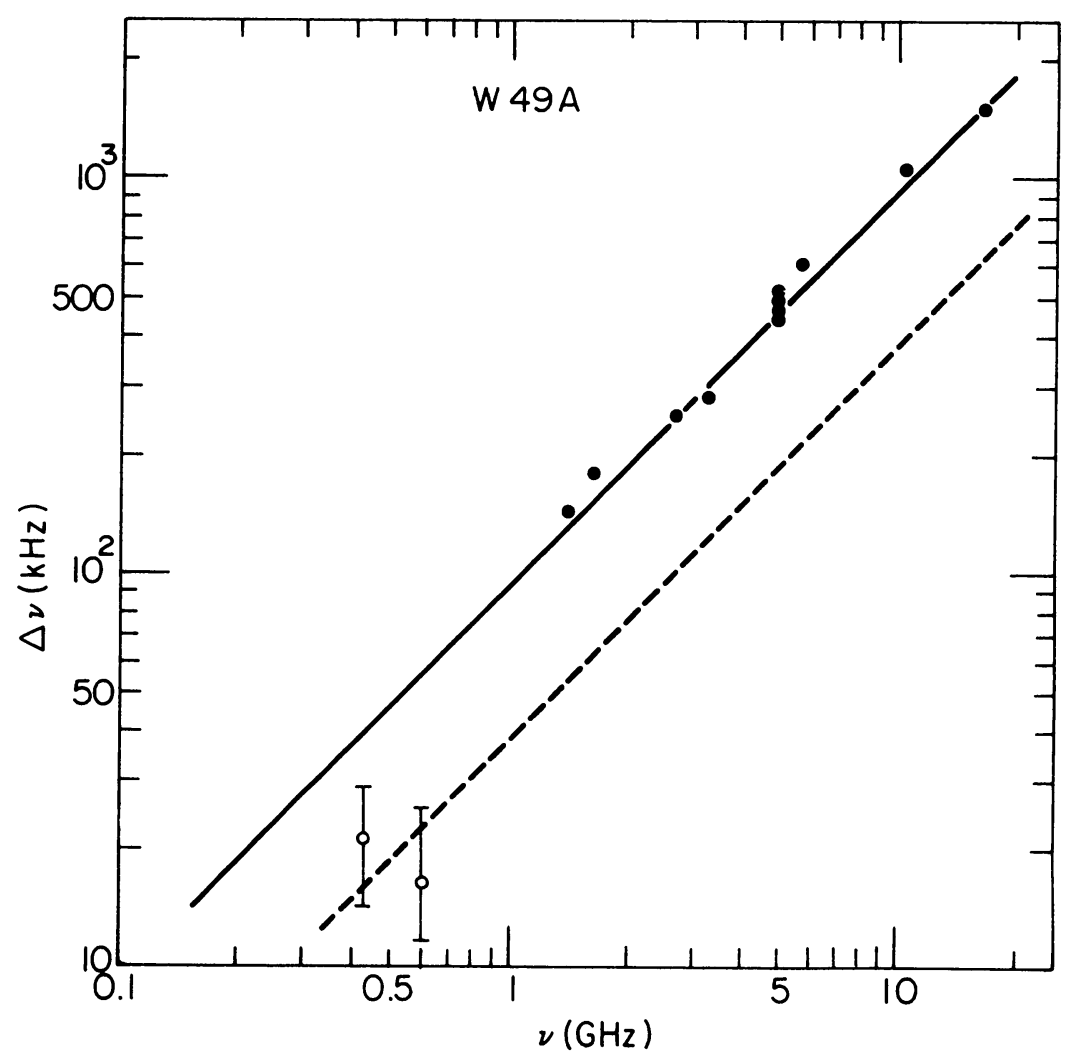

Fig. 4. Widths of Hn $\alpha$ lines from W49A. Open circles indicate present results. Solid and dashed lines represent expected widths of Doppler broadened lines.

energy into the line wings. The resulting lower peak intensity becomes more difficult to detect. A combination of these two factors may explain why pressure broadening effects have not been observed in high quantum level $\alpha$ lines.

In summary, the non-LTE analysis of high frequency hydrogen recombination lines yields physical parameters typical of the most dense structure in the $\mathrm{H}_{\mathrm{II}}$ region. The interpretation of the $H 247,248 \alpha$ lines indicates that they are emitted from the least dense parts of the nebulae $-N_{\mathrm{e}} \lesssim 50 \mathrm{~cm}^{-3}$. These low densities are compatible with the upper limits imposed by pressure broadening considerations.

It appears that a better test of pressure broadening theories is to make observations of a series of higher order lines at nearly the same frequency. This is most practical 
at $v \gtrsim 1.5 \mathrm{GHz}$, and these higher frequency lines are probably emitted from the higher density regions of the nebulae. Higher order observations have been performed by numerous investigators; generally with a negative result on pressure broadening effects. However, recently Simpson (1973) reports that the H211 $\beta$ profiles of Orion A, M17, and W3 are pressure broadened. She also states that the H168 $\beta$ and H211 $\beta$ intensity ratios agree with LTE predictions.

\section{The Cn $\alpha$ Lines}

Current theories of carbon recombination lines predict that under certain conditions the lines should be observed in absorption at some low frequency (Dupree, 1974). However, this has not been observed. In fact for W51 $A$ the carbon line intensity continues to increase relative to hydrogen as the frequency decreases at least down to $0.43 \mathrm{GHz}$.

The W51 $A$ carbon lines are unusual because their observed widths are at least twice as wide as carbon lines in general. The best spectral resolution to date $\left(3.5 \mathrm{~km} \mathrm{~s}^{-1}\right.$, Chaisson, 1973) has not resolved the $\mathrm{Cn} \alpha$ lines from W51 $A$ into more than one component.

An unidentified line was detected in the 247,248 $\alpha$ spectrum of W49 A. That spectrum is displayed in Figure 2. Pankonin et al. (1973) discuss that this is due to electronic recombination onto atomic carbon located in an intervening spiral arm between the nebula $\mathrm{W} 49 A$ and the observer.

With the possible exception of a carbon line toward W $3 A$ (Chaisson, 1972) previous observations have tended to associate the detected carbon recombination emission with $\mathrm{HI}$ clouds which may be physically related to $\mathrm{H}$ II regions. If the present identification is correct, then there are important implications that conditions exist for detectable carbon recombination emission to originate far from normal $\mathrm{HII}$ regions.

\section{Acknowledgements}

The active collaboration of Dr A. Parrish in many phases of the Arecibo recombination line study is gratefully acknowledged. The Arecibo Observatory, National Astronomy and Ionosphere Center, is operated by Cornell University under contract to the U.S. National Science Foundation.

\section{References}

Chaisson, E. J.: 1972, Nature, Phys. Sci. 239, 83.

Chaisson, E. J.: 1973 , in press.

Churchwell, E.: 1970, Ph.D. Thesis, Indiana University.

Dupree, A. K.: 1974, Astrophys. J. 187, 25.

Griem, H. R.: 1967, Astrophys. J. 148, 547.

Palmer, P., Zuckerman, B., Penfield, H., Lilley, A. E., and Mezger, P. G. : 1969, Astrophys. J. 156, 887.

Pankonin, V.: 1973, Ph.D. Thesis, Cornell University.

Pankonin, V. and Parrish, A.: 1974, in preparation. 
Pankonin, V., Parrish, A., and Terzian, Y.: 1973, Astrophys. J. Letters 180, L113.

Parrish, A., Pankonin, V., Heiles, C., Rankin, J. M., and Terzian, Y.: 1972, Astrophys. J. $178,673$.

Parrish, A., Pankonin, V., and Terzian, Y.: 1974, in preparation.

Pedlar, A. and Davies, R. D.: 1972, Monthly Notices Roy. Astron. Soc. 159, 129.

Penfield, H., Palmer, P., and Zuckerman, B.: 1967, Astrophys. J. 148, L25.

Simpson, J. P.: 1973, Astrophys. Space Sci. 20, 187.

\section{Yervant Terzian}

Vernon Pankonin

National Astronomy and Ionosphere Center,

Cornell University,

Ithaca, New York 14850, U.S.A.

\section{DISCUSSION}

Zuckerman: You mentioned the possible detection of the $\mathrm{H} 274 \alpha$ line at Arecibo. I would like to point out that this would set a record in radio astronomy as the lowest frequency spectral line yet observed. Previously the H253 $\alpha$ line observed in 1966 by Penfield, Palmer and myself had the lowest frequency.

Terzian: We have recently made observations of the $\mathrm{H} 274 \alpha$ line at $318 \mathrm{MHz}$, and we have good indications of a detection; however, we need a better signal to noise ratio before we say anything more on this.

Mezger: Apart from breaking an all-time record on quantum number, the thing has serious repercussions on the nature of the carbon recombination line. 